\title{
2 \\ Solving set-valued constraint satisfaction problems
}

\author{
Luc Jaulin \\ ENSIETA \\ 2 rue François Verny, 29200 Brest, France \\ http://www.ensieta.fr/jaulin/ \\ jaulinlu@ensieta.fr
}

\begin{abstract}
In this paper, we consider the resolution of constraint satisfaction problems in the case where the variables of the problem are subsets of $\mathbb{R}^{n}$. In order to use a constraint propagation approach, we introduce sets intervals, which are sets of subsets of $\mathbb{R}^{n}$ with a lower bound and an upper bound with respect to the inclusion. Then, we propose an arithmetic for them. This makes possible to build projection operators that are then used by the propagation. In order to illustrate the principle and the efficiency of the approach, a testcase is provided.
\end{abstract}

\section{Introduction}

Constraint satisfaction problems involving subsets of $\mathbb{R}^{n}$ (namely set-valued constraint satisfaction problems or SVCSP for short) can appear in several engineering applications, typically, when arbitrary shapes (i.e. that cannot be parametrized) are involved. The reconstruction a three dimensional object from photos, mapping an environment from sonar measurements ([12], [16]), or characterizing invariant sets of dynamic systems [2] can be represented by SVCSP. This paper introduces in Section 2 a new type of numbers, namely set intervals, which make possible to use constraint propagation methods for solving SVCSP. In Section 3, an arithmetic for set intervals is proposed. This arithmetic is then used to build projection operators. An illustrative application is provided in Section 4 . Section 5 concludes the paper.

\section{Set intervals}

\subsection{Definition}

Given two sets $\mathbb{A}^{-}$and $\mathbb{A}^{+}$of $\mathbb{R}^{n}$, the pair $\left[\mathbb{A}^{-}, \mathbb{A}^{+}\right]$which encloses all sets $\mathbb{A}$ such that

$$
\mathbb{A}^{-} \subset \mathbb{A} \subset \mathbb{A}^{+}
$$

is a set interval and will be denoted by $[\mathbb{A}]$ (see Figure 1). The set interval $[\emptyset, \emptyset]$ is a singleton which contains a single element: the empty set $\emptyset$. The set interval $\left[\emptyset, \mathbb{R}^{n}\right]$ encloses all sets of $\mathbb{R}^{n}$. If $\mathbb{A}^{-} \not \subset \mathbb{A}^{+}$, then $\left[\mathbb{A}^{-}, \mathbb{A}^{+}\right]$is empty. $A$ set interval is a way to handle and to compute with uncertain sets (see [7]). The 
idea that is developed in this paper follows the foundations of interval analysis that has been built to handle uncertain real numbers [13], [18], to solve realvalued nonlinear problems (see e.g. [5], [14], [8]), or to provide mathematical proofs (see, e.g., [17], [6], [15]).

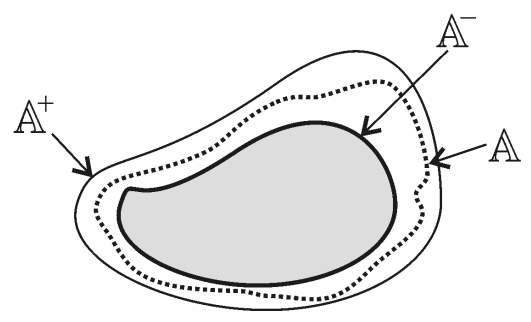

Figure 1: The set $\mathbb{A}$ can be approximated by the set interval $\left[\mathbb{A}^{-}, \mathbb{A}^{+}\right]$

\subsection{Arithmetic}

We shall now define some operations that can be used for set intervals. Two types of operations can be considered.

- Specific set interval operations. Since set intervals are sets of sets (i.e., their elements are sets), the intersection, the union, the inclusion can be defined. In order to avoid any confusion with the operations of their elements, these operations will be denoted in a squared manner (e.g. $\sqcap, \sqcup, \sqsubset$ ).

- Set extension. All operations existing for elements of a set interval (which are sets) such as $\cap, \cup$, reciprocal image , direct image, ... can be extended to set intervals [10].

Let us first start with specific set interval operations.

Intersection. The set interval intersection between two set intervals is defined by

$$
[\mathbb{A}] \sqcap[\mathbb{B}]=\{\mathbb{X}, \mathbb{X} \in[\mathbb{A}] \text { and } \mathbb{X} \in[\mathbb{B}]\}
$$

Since

$$
\begin{aligned}
& \left\{\begin{array} { l } 
{ \mathbb { X } \in [ \mathbb { A } ] } \\
{ \mathbb { X } \in [ \mathbb { B } ] }
\end{array} \Leftrightarrow \left\{\begin{array}{l}
\mathbb{A}^{-} \subset \mathbb{X} \subset \mathbb{A}^{+} \\
\mathbb{B}^{-} \subset \mathbb{X} \subset \mathbb{B}^{+}
\end{array}\right.\right. \\
& \Leftrightarrow \mathbb{A}^{-} \cup \mathbb{B}^{-} \subset \mathbb{X} \subset \mathbb{A}^{+} \cap \mathbb{B}^{+} \Leftrightarrow \mathbb{X} \in\left[\mathbb{A}^{-} \cup \mathbb{B}^{-}, \mathbb{A}^{+} \cap \mathbb{B}^{+}\right],
\end{aligned}
$$

the set interval $[\mathbb{A}] \sqcap[\mathbb{B}]$ is given by

$$
\left[\mathbb{A}^{-}, \mathbb{A}^{+}\right] \sqcap\left[\mathbb{B}^{-}, \mathbb{B}^{+}\right]=\left[\mathbb{A}^{-} \cup \mathbb{B}^{-}, \mathbb{A}^{+} \cap \mathbb{B}^{+}\right] .
$$

Inclusion. We define the set interval inclusion as follows

$$
[\mathbb{A}] \sqsubset[\mathbb{B}] \Leftrightarrow[\mathbb{A}] \sqcap[\mathbb{B}]=[\mathbb{B}] .
$$


Set interval envelope. Consider a collection $\left\{\mathbb{A}_{i}, i \in \mathbb{I}\right\}$ of sets of $\mathbb{R}^{n}$. The set interval envelope $\square\left\{\mathbb{A}_{i}, i \in \mathbb{I}\right\}$ is the smallest set interval (with respect to $\sqsubset)$ enclosing all $\mathbb{A}_{i}, i \in \mathbb{I}$. We have

$$
\square\left\{\mathbb{A}_{i}, i \in \mathbb{I}\right\}=\left[\bigcap_{i \in \mathbb{I}} \mathbb{A}_{i}, \bigcup_{i \in \mathbb{I}} \mathbb{A}_{i}\right] .
$$

For instance,

$$
\square\{[1,4],[3,7],[2,6]\}=[[3,4],[1,7]] .
$$

Union. The set interval union between two set intervals $[\mathbb{A}]$ and $[\mathbb{B}]$ is the smallest set interval which encloses both $[\mathbb{A}]$ and $[\mathbb{B}]$. We have

$$
[\mathbb{A}] \sqcup[\mathbb{B}]=\square\{\mathbb{X}, \mathbb{X} \in[\mathbb{A}] \text { or } \mathbb{X} \in[\mathbb{B}]\} .
$$

It can easily be proven that

$$
[\mathbb{A}] \sqcup[\mathbb{B}]=\left[\mathbb{A}^{-} \cap \mathbb{B}^{-}, \mathbb{A}^{+} \cup \mathbb{B}^{+}\right] .
$$

Set extension of operators. Following the basic idea of Moore [13], it is possible to extend set operations to set intervals. If $\diamond \in\{\cap, \cup, \times, \backslash, \ldots\}$, where $\times$ is the Cartesian product and $\backslash$ is the restriction (or trim) operator. We have

$$
\left[\mathbb{A}^{-}, \mathbb{A}^{+}\right] \diamond\left[\mathbb{B}^{-}, \mathbb{B}^{+}\right]=\square\left\{\mathbb{C}, \mathbb{A} \in\left[\mathbb{A}^{-}, \mathbb{A}^{+}\right], \mathbb{B} \in\left[\mathbb{B}^{-}, \mathbb{B}^{+}\right], \mathbb{C}=\mathbb{A} \diamond \mathbb{B}\right\} .
$$

It is trivial to check, from the monotony of the operators, that

$$
\begin{array}{ll}
\text { (i) } & {\left[\mathbb{A}^{-}, \mathbb{A}^{+}\right] \cap\left[\mathbb{B}^{-}, \mathbb{B}^{+}\right]=\left[\mathbb{A}^{-} \cap \mathbb{B}^{-}, \mathbb{A}^{+} \cap \mathbb{B}^{+}\right]} \\
\text {(ii) }\left[\mathbb{A}^{-}, \mathbb{A}^{+}\right] \cup\left[\mathbb{B}^{-}, \mathbb{B}^{+}\right]=\left[\mathbb{A}^{-} \cup \mathbb{B}^{-}, \mathbb{A}^{+} \cup \mathbb{B}^{+}\right] \\
\text {(iii) }\left[\mathbb{A}^{-}, \mathbb{A}^{+}\right] \times\left[\mathbb{B}^{-}, \mathbb{B}^{+}\right]=\left[\mathbb{A}^{-} \times \mathbb{B}^{-}, \mathbb{A}^{+} \times \mathbb{B}^{+}\right] \\
\text {(iv) }\left[\mathbb{A}^{-}, \mathbb{A}^{+}\right] \backslash\left[\mathbb{B}^{-}, \mathbb{B}^{+}\right]=\left[\mathbb{A}^{-} \backslash \mathbb{B}^{+}, \mathbb{A}^{+} \backslash \mathbb{B}^{-}\right] .
\end{array}
$$

Extension of functions. A set-valued function $f$ can be extended to set intervals as follows

$$
f\left(\left[\mathbb{A}^{-}, \mathbb{A}^{+}\right]\right)=\square\left\{f(\mathbb{A}), \mathbb{A} \in\left[\mathbb{A}^{-}, \mathbb{A}^{+}\right]\right\} .
$$

When $f$ is inclusion monotonic (as it is the case when $f$ is already an extension to sets of vector-valued functions), we have

$$
f\left(\left[\mathbb{A}^{-}, \mathbb{A}^{+}\right]\right)=\left[f\left(\mathbb{A}^{-}\right), f\left(\mathbb{A}^{+}\right)\right] .
$$

Extension of reciprocal functions. Consider a point-valued function $f$. The reciprocal image function $f$ can be extended to set intervals as follows

$$
f^{-1}\left(\left[\mathbb{B}^{-}, \mathbb{B}^{+}\right]\right)=\square\left\{f^{-1}(\mathbb{B}), \mathbb{B} \in\left[\mathbb{B}^{-}, \mathbb{B}^{+}\right]\right\} .
$$

Since $f^{-1}$ is inclusion monotonic, we have

$$
f^{-1}\left(\left[\mathbb{B}^{-}, \mathbb{B}^{+}\right]\right)=\left[f^{-1}\left(\mathbb{B}^{-}\right), f^{-1}\left(\mathbb{B}^{+}\right)\right] .
$$


But another point of view could be adopted for the reciprocal extension. Let us define the reverse set-valued function

$$
f^{\#}(\mathbb{B})=\{\mathbb{A}, f(\mathbb{A})=\mathbb{B}\} .
$$

Let us stress that $f^{\#}$ is not the classical inverse function $f^{-1}$ considered in a set-theoretical context. Its extension to set intervals is

$$
f^{\#}\left(\left[\mathbb{B}^{-}, \mathbb{B}^{+}\right]\right)=\square\left\{\mathbb{A}, f(\mathbb{A}) \in\left[\mathbb{B}^{-}, \mathbb{B}^{+}\right]\right\} .
$$

Unfortunately, due to the multivoque nature of $f^{\#}$ it is often difficult to compute $f^{\#}(\mathbb{B})$. First results, on how to compute $f^{\#}(\mathbb{B})$ can be found in [11]. Let us now give two examples to illustrate this difficulty and the differences between $f^{-1}\left(\left[\mathbb{B}^{-}, \mathbb{B}^{+}\right]\right)$and $f^{\#}\left(\left[\mathbb{B}^{-}, \mathbb{B}^{+}\right]\right)$.

Example 1. Consider the discrete-valued function $f$, defined by Figure 2 . Note that in this paper, for simplicity, the sets that have been considered are subsets of $\mathbb{R}^{n}$, but the approach can be extended to other types of ordered sets, without any difficulty. We have

$$
\begin{array}{ll}
f^{-1}(\mathbb{B}) & =\{a, b, c, e\} \\
f^{\#}(\mathbb{B}) & =\emptyset \\
f^{-1}(\{2,3\}) & =\{a, b, c\} \\
f^{\#}(\{2,3\}) & =\{\{a, b\},\{b, c\},\{a, b, c\},\{a, b, d\},\{b, c, d\},\{a, b, c, d\}\} .
\end{array}
$$

Since, we cannot find a set $\mathbb{Z}$ such that $f(\mathbb{Z})=\mathbb{B}$ implies that $f^{\#}(\mathbb{B})=\emptyset$. We have six sets $\mathbb{X}$ such that $f(\mathbb{X}))=\{1,2\}$ and thus $f^{\#}(\{2,3\})$ contains six elements. Note that $f^{\#}$ is not inclusion monotonic, i.e., we have

$$
\mathbb{X} \subset \mathbb{Y} \nRightarrow f^{\#}(\mathbb{X}) \subset f^{\#}(\mathbb{Y}) .
$$

Take for instance $\mathbb{X}=\{2,3\}$ and $\mathbb{Y}=\mathbb{B}$ as a counterexample. If $f$ is bijective, $f^{\#}$ and $f^{-1}$ are identical. Let us now illustrate the notion of set intervals. We have

$$
\begin{aligned}
& f^{-1}([\{2,3\},\{2,3,4\}])=[\{a, b, c\},\{a, b, c, e\}] \\
& f^{\#}([\{2,3\},\{2,3,4\}])=[\{c\},\{a, b, c, d, e\}] .
\end{aligned}
$$

Example 2. If $f(x)=x^{2}$ and $\mathbb{B}=[4,9]$. We have

$$
f^{-1}([4,9])=[-3,-2] \cup[2,3],
$$

whereas $f^{\#}([4,9])$ contains a infinite number of sets. For instance, the four following sets

$$
[-3,-2] ;[2,3] ;[-3,-2] \cup[2,3] ;[-3,-2.5] \cup[2,2.5]
$$

belong to $f^{\#}([4,9])$. The lower bound of $f^{\#}([4,9])$ with respect to the inclusion is the empty set. If we consider the set interval

$$
\left[\mathbb{B}^{-}, \mathbb{B}^{+}\right]=[[4,9],[1,16]],
$$

we have

$$
\left[\mathbb{A}^{-}, \mathbb{A}^{+}\right]=f^{\#}\left(\left[\mathbb{B}^{-}, \mathbb{B}^{+}\right]\right)=[\underbrace{\emptyset}_{=\mathbb{A}^{-}}, \underbrace{[-4,-1] \cup[1,4]}_{=\mathbb{A}^{+}}]
$$




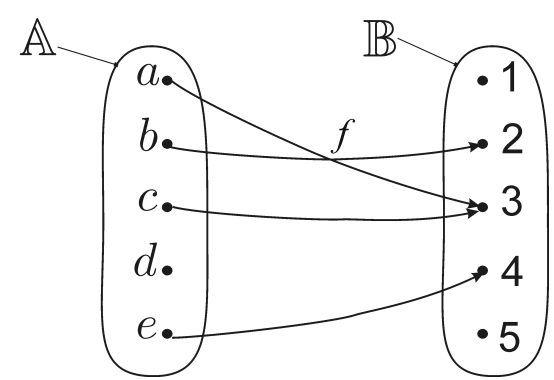

Figure 2: Function to be inverted

\subsection{Natural set interval extension}

Consider a set-valued expression $f\left(\mathbb{X}_{1}, \mathbb{X}_{2}, \ldots, \mathbb{X}_{n}\right)$ made with operators and functions already defined for the $\mathbb{X}_{i}$ 's (such as $+, \cap, \cup, \ldots$ ). The natural setinterval extension $[f]$ of $f$ is the set interval function whose expressions is obtained by taking that of $f$ and by replacing all sets $\mathbb{X}_{i}$ by set intervals $\left[\mathbb{X}_{i}\right]$ and all operators and elementary functions involved in $f$ by their set-interval counterparts. For instance, the natural set interval extension associated with the set expression

$$
f\left(\mathbb{X}_{1}, \mathbb{X}_{2}, \mathbb{X}_{3}\right)=\mathbb{X}_{1} \cup\left(\mathbb{X}_{2} \cap g\left(\mathbb{X}_{3}\right)\right)
$$

is

$$
[f]\left(\left[\mathbb{X}_{1}\right],\left[\mathbb{X}_{2}\right],\left[\mathbb{X}_{3}\right]\right)=\left[\mathbb{X}_{1}\right] \cup\left(\left[\mathbb{X}_{2}\right] \cap g\left(\left[\mathbb{X}_{3}\right]\right)\right) .
$$

Theorem 1. Consider an expression $f\left(\mathbb{X}_{1}, \mathbb{X}_{2}, \ldots, \mathbb{X}_{n}\right)$ made with operators and functions already defined for the $\mathbb{X}_{i}$ 's. If $\mathbb{X}_{1} \in\left[\mathbb{X}_{1}\right], \ldots, \mathbb{X}_{n} \in\left[\mathbb{X}_{n}\right]$ then

$$
f\left(\mathbb{X}_{1}, \mathbb{X}_{2}, \ldots, \mathbb{X}_{n}\right) \in[f]\left(\left[\mathbb{X}_{1}\right],\left[\mathbb{X}_{2}\right], \ldots,\left[\mathbb{X}_{n}\right]\right)
$$

Moreover, if in the expression of $f$, all $\mathbb{X}_{i}$ occur only once, the set interval evaluation is minimal with respect to the inclusion.

Proof. See [9], Section 2.2.3

Example 3. Using Theorem 1, if $\mathbb{A} \in[\mathbb{A}], \mathbb{B} \in[\mathbb{B}]$, then

$$
(\mathbb{A} \cup \mathbb{B}) \backslash(\mathbb{A} \cap \mathbb{B}) \in([\mathbb{A}] \cup[\mathbb{B}]) \backslash([\mathbb{A}] \cap[\mathbb{B}]) .
$$

Take for instance

$$
\begin{aligned}
{\left[\mathbb{A}^{-}, \mathbb{A}^{+}\right] } & =[[1,3],[0,4]] \\
{\left[\mathbb{B}^{-}, \mathbb{B}^{+}\right] } & =[[2,5],[1,6]] .
\end{aligned}
$$

Since

$$
\begin{aligned}
([\mathbb{A}] \cup[\mathbb{B}]) \backslash([\mathbb{A}] \cap[\mathbb{B}]) & =\left[\mathbb{A}^{-} \cup \mathbb{B}^{-}, \mathbb{A}^{+} \cup \mathbb{B}^{+}\right] \backslash\left[\mathbb{A}^{-} \cap \mathbb{B}^{-}, \mathbb{A}^{+} \cap \mathbb{B}^{+}\right] \\
& =\left[\left(\mathbb{A}^{-} \cup \mathbb{B}^{-}\right) \backslash\left(\mathbb{A}^{+} \cap \mathbb{B}^{+}\right),\left(\mathbb{A}^{+} \cup \mathbb{B}^{+}\right) \backslash\left(\mathbb{A}^{-} \cap \mathbb{B}^{-}\right)\right],
\end{aligned}
$$


we have

$$
\begin{aligned}
& {\left[\left(\mathbb{A}^{-} \cup \mathbb{B}^{-}\right) \backslash\left(\mathbb{A}^{+} \cap \mathbb{B}^{+}\right),\left(\mathbb{A}^{+} \cup \mathbb{B}^{+}\right) \backslash \mathbb{A}^{-} \cap \mathbb{B}^{-}\right] } \\
= & {[([1,3] \cup[2,5]) \backslash([0,4] \cap[1,6]),([0,4] \cup[1,6]) \backslash([1,3] \cap[2,5])] } \\
= & {[[1,5] \backslash[1,4],[0,6] \backslash[2,3]] } \\
= & {[[4,5],[0,2] \cup[3,6]] . }
\end{aligned}
$$

Dependency problem. As it is the case for interval arithmetic, the dependency problem also exists for set intervals. For instance,

$$
\left[\mathbb{A}^{-}, \mathbb{A}^{+}\right] \backslash\left[\mathbb{A}^{-}, \mathbb{A}^{+}\right]=\left[\mathbb{A}^{-} \backslash \mathbb{A}^{+}, \mathbb{A}^{+} \backslash \mathbb{A}^{-}\right]=\left[\emptyset, \mathbb{A}^{+} \backslash \mathbb{A}^{-}\right]
$$

Of course, we have the inclusion property

$$
\left\{\mathbb{A} \backslash \mathbb{A}, \mathbb{A} \in\left[\mathbb{A}^{-}, \mathbb{A}^{+}\right]\right\}=[\emptyset, \emptyset] \sqsubset\left[\emptyset, \mathbb{A}^{+} \backslash \mathbb{A}^{-}\right]
$$

but the resulting set interval is not minimal.

Example 4. Consider two equivalent expressions of the exclusive union

$$
\begin{aligned}
& f(\mathbb{A}, \mathbb{B})=(\mathbb{A} \backslash \mathbb{B}) \cup(\mathbb{B} \backslash \mathbb{A}) \\
& g(\mathbb{A}, \mathbb{B})=(\mathbb{A} \cup \mathbb{B}) \backslash(\mathbb{A} \cap \mathbb{B}) .
\end{aligned}
$$

The two natural set interval extension are given by

$$
\begin{aligned}
& {[f]([\mathbb{A}],[\mathbb{B}])=([\mathbb{A}] \backslash[\mathbb{B}]) \cup([\mathbb{B}] \backslash[\mathbb{A}])} \\
& {[g]([\mathbb{A}],[\mathbb{B}])=([\mathbb{A}] \cup[\mathbb{B}]) \backslash([\mathbb{A}] \cap[\mathbb{B}]) .}
\end{aligned}
$$

Let us evaluate these two expressions for the two set intervals $[\mathbb{A}],[\mathbb{B}]$ represented on subfigures (a) and (b) of Figure $3^{1}$. Subfigures (c),(d), (e) represent $[\mathbb{A}] \backslash[\mathbb{B}],[\mathbb{B}] \backslash[\mathbb{A}],([\mathbb{A}] \backslash[\mathbb{B}]) \cup([\mathbb{B}] \backslash[\mathbb{A}])$, respectively. Subfigures $(\mathrm{d}),(\mathrm{g}),(\mathrm{h})$ represent $([\mathbb{A}] \cup[\mathbb{B}]),([\mathbb{A}] \cap[\mathbb{B}]),([\mathbb{A}] \cup[\mathbb{B}]) \backslash([\mathbb{A}] \cap[\mathbb{B}])$, respectively. For this example, the two natural set interval extensions provide similar results, but it is not the case in general.

\section{$3 \quad$ Projection operators}

Contractors are powerful tools to solve efficiently CSP [19], [4], [1]. They are based on the notion of constraint projection [3] that will now be considered in the context of set-valued constraints. Consider a SVCSP

$$
\left\{\begin{array}{c}
\mathcal{R}\left(\mathbb{X}_{1}, \ldots, \mathbb{X}_{p}\right) \\
\mathbb{X}_{1} \in\left[\mathbb{X}_{1}\right], \ldots \mathbb{X}_{p} \in\left[\mathbb{X}_{p}\right]
\end{array}\right.
$$

\footnotetext{
${ }^{1}$ Color code. For the graphical representation of a set interval $[\mathbb{X}]=\left[\mathbb{X}^{-}, \mathbb{X}^{+}\right]$, the black boxes are inside $\mathbb{X}^{-}$, the grey boxes are outside $\mathbb{X}^{+}$and the white boxes are inside $\mathbb{X}^{+}$and outside $\mathbb{X}^{-}$.
} 

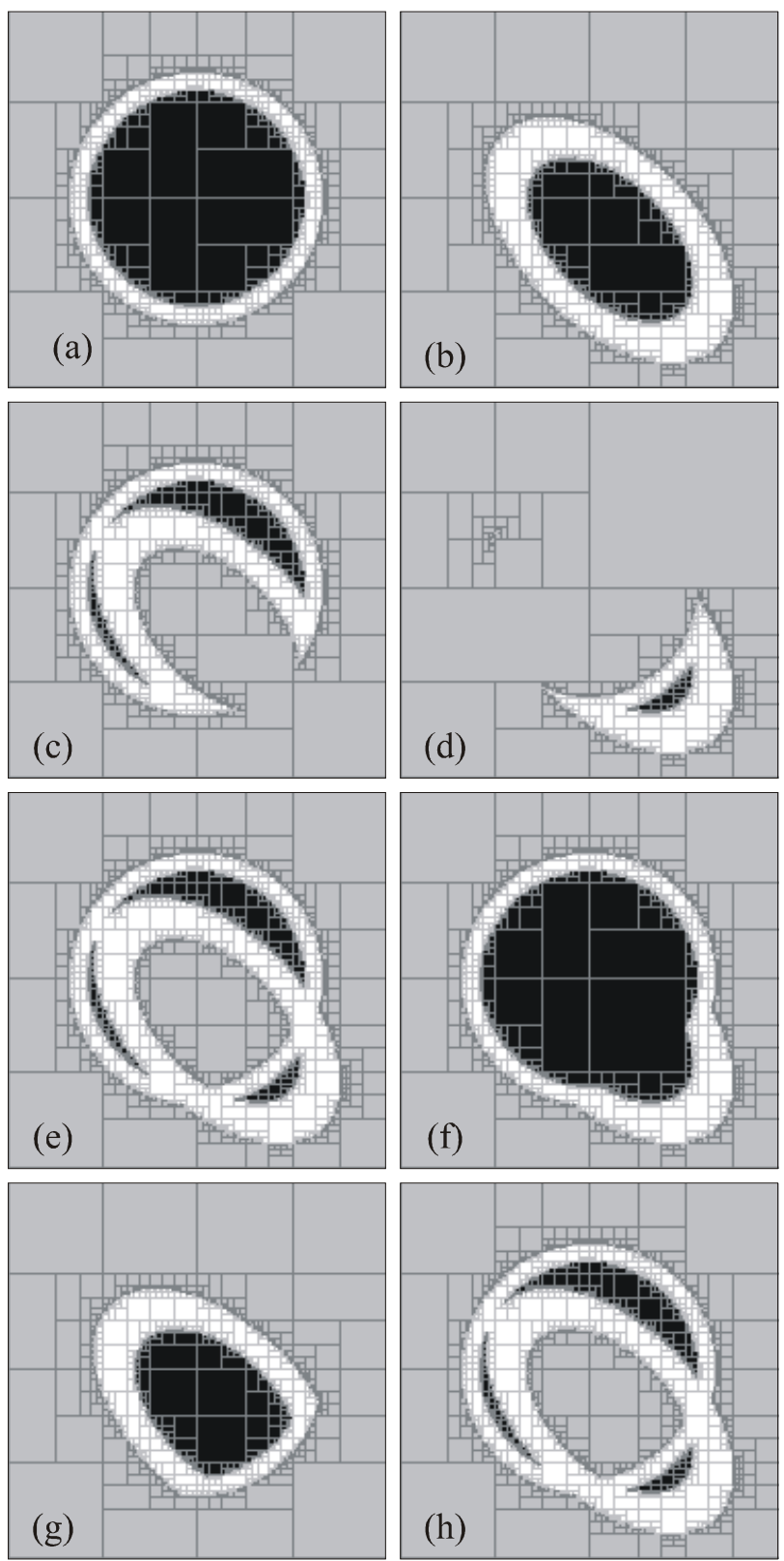

Figure 3: Illustration of the set interval arithmetic 
Projecting the relation (or the constraint) $\mathcal{R}$ with respect to $\mathbb{X}_{1}\left(\right.$ resp. $\mathbb{X}_{2}, \ldots$ ) amounts to computing the smallest set interval which encloses all feasible $\mathbb{X}_{1}$ $\left(\right.$ resp. $\left.\mathbb{X}_{2}, \ldots\right)$. In a formal way, the first projection can be defined as

$\pi_{1}\left(\mathcal{R},\left[\mathbb{X}_{1}\right], \ldots,\left[\mathbb{X}_{p}\right]\right)=\square\left\{\mathbb{X}_{1} \in\left[\mathbb{X}_{1}\right], \exists \mathbb{X}_{2} \in\left[\mathbb{X}_{2}\right], \ldots, \exists \mathbb{X}_{p} \in\left[\mathbb{X}_{p}\right], \mathcal{R}\left(\mathbb{X}_{1}, \ldots, \mathbb{X}_{p}\right)\right\}$.

The following theorems shows how such a projection can be performed for some particular primitive set-valued constraints.

Theorem 2. Consider the SVCSP

$$
\left\{\begin{array}{c}
\mathbb{A} \subset \mathbb{B} \\
\mathbb{A} \in[\mathbb{A}], \mathbb{B} \in[\mathbb{B}],
\end{array}\right.
$$

where $[\mathbb{A}],[\mathbb{B}]$ are set intervals. The projection with respect to $\mathbb{A}, \mathbb{B}$ is given by the following operations.

$$
\begin{cases}\text { (i) } & {[\mathbb{A}]:=[\mathbb{A}] \sqcap([\mathbb{A}] \cap[\mathbb{B}])} \\ \text { (ii) } & {[\mathbb{B}]:=[\mathbb{B}] \sqcap([\mathbb{A}] \cup[\mathbb{B}])}\end{cases}
$$

or equivalently

$$
\begin{cases}\text { (i) } & {[\mathbb{A}]:=\left[\mathbb{A}^{-}, \mathbb{A}^{+} \cap \mathbb{B}^{+}\right]} \\ \text {(ii) } & {[\mathbb{B}]:=\left[\mathbb{B}^{-} \cup \mathbb{A}^{-}, \mathbb{B}^{+}\right] .}\end{cases}
$$

Proof. Let us first prove (i). We have

$$
\left\{\begin{array} { c } 
{ \mathbb { A } \subset \mathbb { B } } \\
{ \mathbb { A } \in [ \mathbb { A } ] } \\
{ \mathbb { B } \in [ \mathbb { B } ] }
\end{array} \Leftrightarrow \left\{\begin{array} { c } 
{ \mathbb { A } = \mathbb { A } \cap \mathbb { B } } \\
{ \mathbb { A } \in [ \mathbb { A } ] } \\
{ \mathbb { B } \in [ \mathbb { B } ] }
\end{array} \Leftrightarrow \left\{\begin{array}{c}
\mathbb{A} \in[\mathbb{A}] \sqcap([\mathbb{A}] \cap[\mathbb{B}]) \\
\mathbb{B} \in[\mathbb{B}]
\end{array}\right.\right.\right.
$$

Now

$$
\begin{aligned}
& {[\mathbb{A}] \sqcap([\mathbb{A}] \cap[\mathbb{B}]) \stackrel{(2, \mathrm{i})}{=} \quad\left[\mathbb{A}^{-}, \mathbb{A}^{+}\right] \sqcap\left[\mathbb{A}^{-} \cap \mathbb{B}^{-}, \mathbb{A}^{+} \cap \mathbb{B}^{+}\right]} \\
& \stackrel{(1)}{=} \quad\left[\mathbb{A}^{-} \cup\left(\mathbb{A}^{-} \cap \mathbb{B}^{-}\right), \mathbb{B}^{+} \cap \mathbb{A}^{+} \cap \mathbb{B}^{+}\right] \\
& =\left[\mathbb{A}^{-}, \mathbb{A}^{+} \cap \mathbb{B}^{+}\right] \text {. }
\end{aligned}
$$

Let us now prove (ii). We have

$$
\left\{\begin{array} { c } 
{ \mathbb { A } \subset \mathbb { B } } \\
{ \mathbb { A } \in [ \mathbb { A } ] } \\
{ \mathbb { B } \in [ \mathbb { B } ] }
\end{array} \Leftrightarrow \left\{\begin{array} { c } 
{ \mathbb { B } = \mathbb { A } \cup \mathbb { B } } \\
{ \mathbb { A } \in [ \mathbb { A } ] } \\
{ \mathbb { B } \in [ \mathbb { B } ] }
\end{array} \Leftrightarrow \left\{\begin{array}{c}
\mathbb{B} \in[\mathbb{B}] \sqcap([\mathbb{A}] \cup[\mathbb{B}]) \\
\mathbb{A} \in[\mathbb{A}] .
\end{array}\right.\right.\right.
$$

Now

$$
\begin{array}{rll}
{[\mathbb{B}] \sqcap([\mathbb{B}] \cup[\mathbb{A}])} & \stackrel{(2, \mathrm{ii})}{=} & {\left[\mathbb{B}^{-}, \mathbb{B}^{+}\right] \sqcap\left[\mathbb{A}^{-} \cup \mathbb{B}^{-}, \mathbb{A}^{+} \cup \mathbb{B}^{+}\right]} \\
& \stackrel{(1)}{=} & {\left[\mathbb{B}^{-} \cup \mathbb{A}^{-} \cup \mathbb{B}^{-}, \mathbb{B}^{+} \cap\left(\mathbb{A}^{+} \cup \mathbb{B}^{+}\right)\right]} \\
& = & {\left[\mathbb{A}^{-} \cup \mathbb{B}^{-}, \mathbb{B}^{+}\right] .}
\end{array}
$$

Theorem 3. Consider the SVCSP

$$
\left\{\begin{array}{c}
\mathbb{A} \cap \mathbb{B}=\emptyset \\
\mathbb{A} \in[\mathbb{A}], \mathbb{B} \in[\mathbb{B}]
\end{array}\right.
$$


where $[\mathbb{A}],[\mathbb{B}]$ are set intervals. The projection with respect to $\mathbb{A}, \mathbb{B}$ is given by the following operations

$$
\begin{cases}\text { (i) } & {[\mathbb{A}]:=[\mathbb{A}] \sqcap\left(\left[\emptyset, \mathbb{R}^{n}\right] \backslash[\mathbb{B}]\right)} \\ \text { (ii) } & {[\mathbb{B}]:=[\mathbb{B}] \sqcap\left(\left[\emptyset, \mathbb{R}^{n}\right] \backslash[\mathbb{A}]\right)}\end{cases}
$$

or equivalently

$$
\begin{cases}\text { (i) } & {[\mathbb{A}]:=\left[\mathbb{A}^{-}, \mathbb{A}^{+} \backslash \mathbb{B}^{-}\right]} \\ \text {(ii) } & {[\mathbb{B}]:=\left[\mathbb{B}^{-}, \mathbb{B}^{+} \backslash \mathbb{A}^{-}\right]}\end{cases}
$$

Proof. We shall thus limit ourselves to proving (i), proving (ii) is similar. Given $\mathbb{B}$ we have the following equivalence

$$
\mathbb{A} \cap \mathbb{B}=\emptyset \Leftrightarrow \exists \mathbb{Z} \in\left[\emptyset, \mathbb{R}^{n}\right] \text { such that } \mathbb{A}=\mathbb{Z} \backslash \mathbb{B} \text {. }
$$

Since $\mathbb{A} \in[\mathbb{A}], \mathbb{B} \in[\mathbb{B}], \mathbb{Z} \in[\emptyset, \mathbb{R}]$, using the set interval arithmetic, we get that

$$
\mathbb{Z} \backslash \mathbb{B} \in([\emptyset, \mathbb{R}] \backslash[\mathbb{B}])
$$

and thus

$$
\mathbb{A} \in[\mathbb{A}] \sqcap([\emptyset, \mathbb{R}] \backslash[\mathbb{B}]) .
$$

Using the set interval arithmetic, we get

$$
\begin{array}{ll} 
& {[\mathbb{A}] \sqcap([\emptyset, \mathbb{R}] \backslash[\mathbb{B}])} \\
= & {\left[\mathbb{A}^{-}, \mathbb{A}^{+}\right] \sqcap\left(\left[\emptyset, \mathbb{R}^{n}\right] \backslash\left[\mathbb{B}^{-}, \mathbb{B}^{+}\right]\right)} \\
\stackrel{(2, \text { iv })}{=} & {\left[\mathbb{A}^{-}, \mathbb{A}^{+}\right] \sqcap\left(\left[\emptyset \backslash \mathbb{B}^{+}, \mathbb{R}^{n} \backslash \mathbb{B}^{-}\right]\right)} \\
\stackrel{(1)}{=} & {\left[\mathbb{A}^{-}, \mathbb{A}^{+} \cap \mathbb{R}^{n} \backslash \mathbb{B}^{-}\right]} \\
= & {\left[\mathbb{A}^{-}, \mathbb{A}^{+} \backslash \mathbb{B}^{-}\right] .}
\end{array}
$$

Theorem 4. Consider the SVCSP

$$
\left\{\begin{array}{c}
\mathbb{A} \cap \mathbb{B}=\mathbb{C} \\
\mathbb{A} \in[\mathbb{A}], \mathbb{B} \in[\mathbb{B}], \mathbb{C} \in[\mathbb{C}],
\end{array}\right.
$$

where $[\mathbb{A}],[\mathbb{B}],[\mathbb{C}]$ are set intervals. The projection with respect to $\mathbb{A}, \mathbb{B}, \mathbb{C}$ is given by the following operations

$$
\begin{cases}\text { (i) } & {[\mathbb{C}]:=[\mathbb{C}] \sqcap([\mathbb{A}] \cap[\mathbb{B}])} \\ \text { (ii) } & {[\mathbb{A}]:=[\mathbb{A}] \sqcap\left([\mathbb{C}] \cup\left(\left[\emptyset, \mathbb{R}^{n}\right] \backslash([\mathbb{B}] \backslash[\mathbb{C}])\right)\right)} \\ \text { (iii) } & {[\mathbb{B}]:=[\mathbb{B}] \sqcap\left([\mathbb{C}] \cup\left(\left[\emptyset, \mathbb{R}^{n}\right] \backslash([\mathbb{A}] \backslash[\mathbb{C}])\right)\right)}\end{cases}
$$

or equivalently

$$
\begin{cases}\text { (i) } & {[\mathbb{C}]:=\left[\mathbb{C}^{-} \cup\left(\mathbb{A}^{-} \cap \mathbb{B}^{-}\right), \mathbb{C}^{+} \cap \mathbb{A}^{+} \cap \mathbb{B}^{+}\right]} \\ \text {(ii) } & {[\mathbb{A}]:=\left[\mathbb{A}^{-} \cup \mathbb{C}^{-}, \mathbb{A}^{+} \backslash\left(\mathbb{B}^{-} \backslash \mathbb{C}^{+}\right)\right]} \\ \text {(iii) } & {[\mathbb{B}]:=\left[\mathbb{B}^{-} \cup \mathbb{C}^{-}, \mathbb{B}^{+} \backslash\left(\mathbb{A}^{-} \backslash \mathbb{C}^{+}\right)\right]}\end{cases}
$$

An illustration is represented on Figure 4. Subfigure (a) represents the initial set intervals $[\mathbb{A}],[\mathbb{B}],[\mathbb{C}]$, before projection. These set intervals can be 

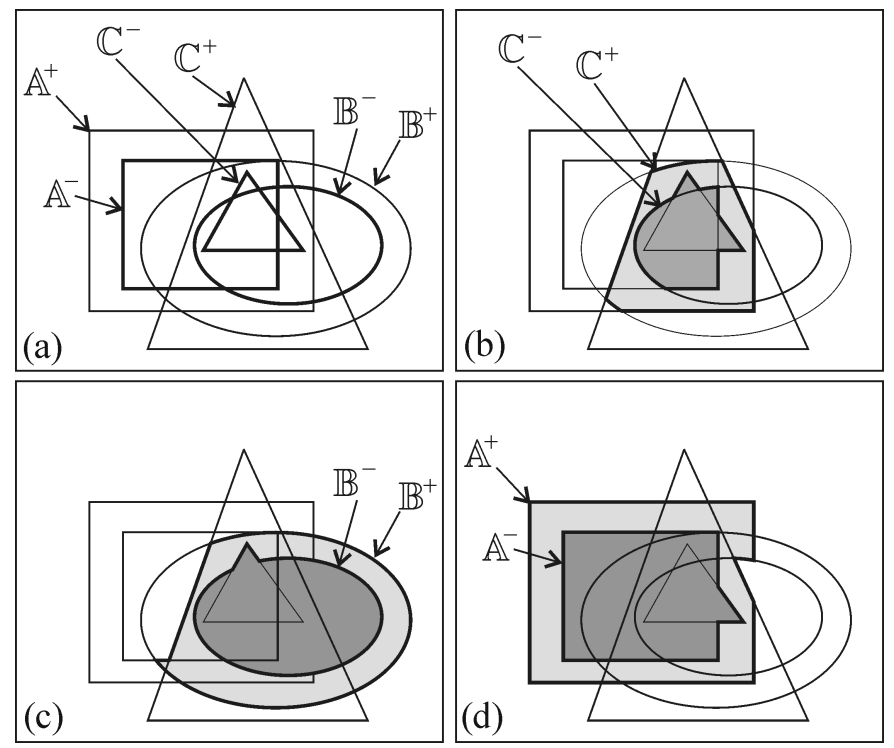

Figure 4: Contraction operator on set intervals associated with the relation $\mathbb{A} \cap \mathbb{B}=\mathbb{C}$

contracted without removing any feasible set, as illustrated by the Figure 4 (b), (c), (d).

Lemma. Before giving the proof, let us recall the two following set equalities.

$$
\begin{array}{ll}
\text { (a) } & \mathbb{B} \cup \mathbb{R}^{n} \backslash(\mathbb{A} \backslash \mathbb{B})=\mathbb{R}^{n} \backslash(\mathbb{A} \backslash \mathbb{B}) \\
\text { (b) } & \mathbb{A} \cap \mathbb{R}^{n} \backslash(\mathbb{B} \backslash \mathbb{C})=\mathbb{A} \backslash(\mathbb{B} \backslash \mathbb{C}) .
\end{array}
$$

Proof. The proof for (i) is trivial. The proof for (iii) is similar than that of (ii). We shall thus limit ourselves to proving (ii). Given $\mathbb{B}, \mathbb{C}$ we have the following equivalence

$$
\mathbb{A} \cap \mathbb{B}=\mathbb{C} \Leftrightarrow \exists \mathbb{Z} \in\left[\emptyset, \mathbb{R}^{n}\right] \text { such that } \mathbb{A}=\mathbb{C} \cup \mathbb{Z} \backslash(\mathbb{B} \backslash \mathbb{C}) .
$$

Since $\mathbb{A} \in[\mathbb{A}], \mathbb{B} \in[\mathbb{B}], \mathbb{C} \in[\mathbb{C}], \mathbb{Z} \in[\emptyset, \mathbb{R}]$, using the set interval arithmetic, we get that

$$
\mathbb{C} \cup \mathbb{Z} \backslash(\mathbb{B} \backslash \mathbb{C}) \in([\mathbb{C}] \cup([\emptyset, \mathbb{R}] \backslash([\mathbb{B}] \backslash[\mathbb{C}])))
$$

and thus

$$
\mathbb{A} \in[\mathbb{A}] \sqcap([\mathbb{C}] \cup([\emptyset, \mathbb{R}] \backslash([\mathbb{B}] \backslash[\mathbb{C}]))) .
$$


Using the set interval arithmetic, we get

$$
\begin{array}{ll} 
& {[\mathbb{A}] \sqcap([\mathbb{C}] \cup([\emptyset, \mathbb{R}] \backslash([\mathbb{B}] \backslash[\mathbb{C}])))} \\
= & {\left[\mathbb{A}^{-}, \mathbb{A}^{+}\right] \sqcap\left(\left[\mathbb{C}^{-}, \mathbb{C}^{+}\right] \cup(\left[\emptyset, \mathbb{R}^{n}\right] \backslash \underbrace{\left(\left[\mathbb{B}^{-}, \mathbb{B}^{+}\right] \backslash\left[\mathbb{C}^{-}, \mathbb{C}^{+}\right]\right)}_{=\left[\mathbb{B}^{-} \backslash \mathbb{C}^{+}, \mathbb{B}^{+} \backslash \mathbb{C}^{-}\right]})\right)} \\
= & {\left[\mathbb{A}^{-}, \mathbb{A}^{+}\right] \sqcap\left(\left[\mathbb{C}^{-}, \mathbb{C}^{+}\right] \cup[\underbrace{\emptyset \backslash \mathbb{B}^{+} \backslash \mathbb{C}^{-}}_{=\emptyset}, \mathbb{R}^{n} \backslash\left(\mathbb{B}^{-} \backslash \mathbb{C}^{+}\right)]\right)} \\
= & {\left[\mathbb{A}^{-}, \mathbb{A}^{+}\right] \sqcap\left(\left[\mathbb{C}^{-}, \mathbb{C}^{+} \cup \mathbb{R}^{n} \backslash\left(\mathbb{B}^{-} \backslash \mathbb{C}^{+}\right)\right]\right)} \\
\stackrel{(4, \mathrm{a})}{=} & {\left[\mathbb{A}^{-}, \mathbb{A}^{+}\right] \sqcap\left(\left[\mathbb{C}^{-}, \mathbb{R}^{n} \backslash\left(\mathbb{B}^{-} \backslash \mathbb{C}^{+}\right)\right]\right)} \\
= & {\left[\mathbb{A}^{-} \cup \mathbb{C}^{-}, \mathbb{A}^{+} \cap \mathbb{R}^{n} \backslash\left(\mathbb{B}^{-} \backslash \mathbb{C}^{+}\right)\right]} \\
\stackrel{(4, \mathrm{~b})}{=} & {\left[\mathbb{A}^{-} \cup \mathbb{C}^{-}, \mathbb{A}^{+} \backslash\left(\mathbb{B}^{-} \backslash \mathbb{C}^{+}\right)\right] .}
\end{array}
$$

Theorem 5. Consider the SVCSP

$$
\left\{\begin{array}{c}
f(\mathbb{A})=\mathbb{B} \\
\mathbb{A} \in[\mathbb{A}], \mathbb{B} \in[\mathbb{B}]
\end{array}\right.
$$

where $[\mathbb{A}],[\mathbb{B}]$ are set intervals. Projections with respect to $\mathbb{A}, \mathbb{B}$ are obtained by the following operations.

$$
\begin{cases}\text { (i) } & {[\mathbb{B}]:=[\mathbb{B}] \sqcap f([\mathbb{A}])} \\ \text { (ii) } & {[\mathbb{A}]:=[\mathbb{A}] \sqcap f^{\#}([\mathbb{B}]) .}\end{cases}
$$

For (i), we could write

$$
\text { (i) } \quad \mathbb{B}^{-}:=\mathbb{B}^{-} \cup f\left(\mathbb{A}^{-}\right) ; \quad \mathbb{B}^{+}=\mathbb{B}^{+} \cap f\left(\mathbb{A}^{+}\right) .
$$

For (ii), due to the non monotonicity of $f^{\#}$, no such relation can be obtained, except for particular cases, such as when $f$ is bijective.

Proof. Let us first prove (i). We have

$$
\left\{\begin{array} { c } 
{ f ( \mathbb { A } ) = \mathbb { B } } \\
{ \mathbb { A } \in [ \mathbb { A } ] } \\
{ \mathbb { B } \in [ \mathbb { B } ] }
\end{array} \Leftrightarrow \left\{\begin{array}{c}
\mathbb{A} \in[\mathbb{A}] \sqcap f^{\#}([\mathbb{B}]) \\
\mathbb{B} \in[\mathbb{B}] \sqcap f([\mathbb{A}]) .
\end{array}\right.\right.
$$

Now

$$
\begin{aligned}
{[\mathbb{B}] \sqcap f([\mathbb{A}]) } & =\left[\mathbb{B}^{-}, \mathbb{B}^{+}\right] \sqcap\left[f\left(\mathbb{A}^{-}\right), f\left(\mathbb{A}^{+}\right)\right] \\
& \stackrel{(1)}{=}\left[\mathbb{B}^{-} \cup f\left(\mathbb{A}^{-}\right), \mathbb{B}^{+} \cap f\left(\mathbb{A}^{+}\right)\right]
\end{aligned}
$$

\section{Test-case}

Consider the following SVCSP

$$
\left\{\begin{array}{cc}
\text { (i) } & \mathbb{X} \subset \mathbb{A} \\
\text { (ii) } & \mathbb{B} \subset \mathbb{X} \\
\text { (iii) } & \mathbb{X} \cap \mathbb{C}=\emptyset \\
\text { (iv) } & f(\mathbb{X})=\mathbb{X}
\end{array}\right.
$$


where $\mathbb{X}$ is an unknown subset of $\mathbb{R}^{2}, f$ is a rotation of $\mathbb{R}^{2}$ around $\mathbf{0}$ with an angle $-\frac{\pi}{6}$, and

$$
\left\{\begin{array}{l}
\mathbb{A}=\left\{\left(x_{1}, x_{2}\right), x_{1}^{2}+x_{2}^{2} \leq 3\right\} \\
\mathbb{B}=\left\{\left(x_{1}, x_{2}\right),\left(x_{1}-0.5\right)^{2}+x_{2}^{2} \leq 0.3\right\} \\
\mathbb{C}=\left\{\left(x_{1}, x_{2}\right),\left(x_{1}-1\right)^{2}+\left(x_{2}-1\right)^{2} \leq 0.15\right\}
\end{array}\right.
$$

In our context, a constraint propagation approach consists in projecting all setvalued constraints several times until no more significant contraction can be observed. Figure 5 illustrates the propagation process (the color signification for subpavings is similar to that of Figure 3). Subfigures (a), (b), (c) represent $\mathbb{A}, \mathbb{B}, \mathbb{C}$. Subfigure $(\mathrm{d})$ represents the set interval $[\mathbb{X}]$ after the projection of constraint (i). If we now project the constraint (ii), we get Subfigure (e) for $[\mathbb{X}]$. Constraint (iii) yields Subfigure (f). Another projection of all four constraints produces Subfigure (f). Finally, Subfigure (g) represents the fixed point that is obtained for $[\mathbb{X}]$.

\section{Conclusion}

This paper proposes to extend the class of problems that can be solved using constraint propagation to set-valued constraint satisfaction problems (SVCSP). The variables of such CSP are subsets of $\mathbb{R}^{n}$ that can be bracketed by pairs of sets. These pairs, named set intervals, form the domains on which the set variables should belong. An arithmetic is provided for set-intervals which make possible to build projection operators and consequently to allow a resolution based on constraint propagation. An illustrative example has been provided to illustrate the principle of the approach.

\section{References}

[1] I. Araya, B. Neveu, and G. Trombettoni. Exploiting common subexpressions in numerical csps. In Proc. CP, Constraint Programming, page 342Ü357, LNCS 5202, 2008.

[2] J. Aubin and H. Frankowska. Set-Valued Analysis. Birkhäuser, Boston, 1990.

[3] F. Benhamou, F. Goualard, L. Granvilliers, and J. F. Puget. Revising hull and box consistency. In Proceedings of the International Conference on Logic Programming, pages 230-244, Las Cruces, NM, 1999.

[4] G. Chabert and L. Jaulin. QUIMPER, A Language for Quick Interval Modelling and Programming in a Bounded-Error Context. Artificial Intelligence, 173:1079-1100, 2009. 

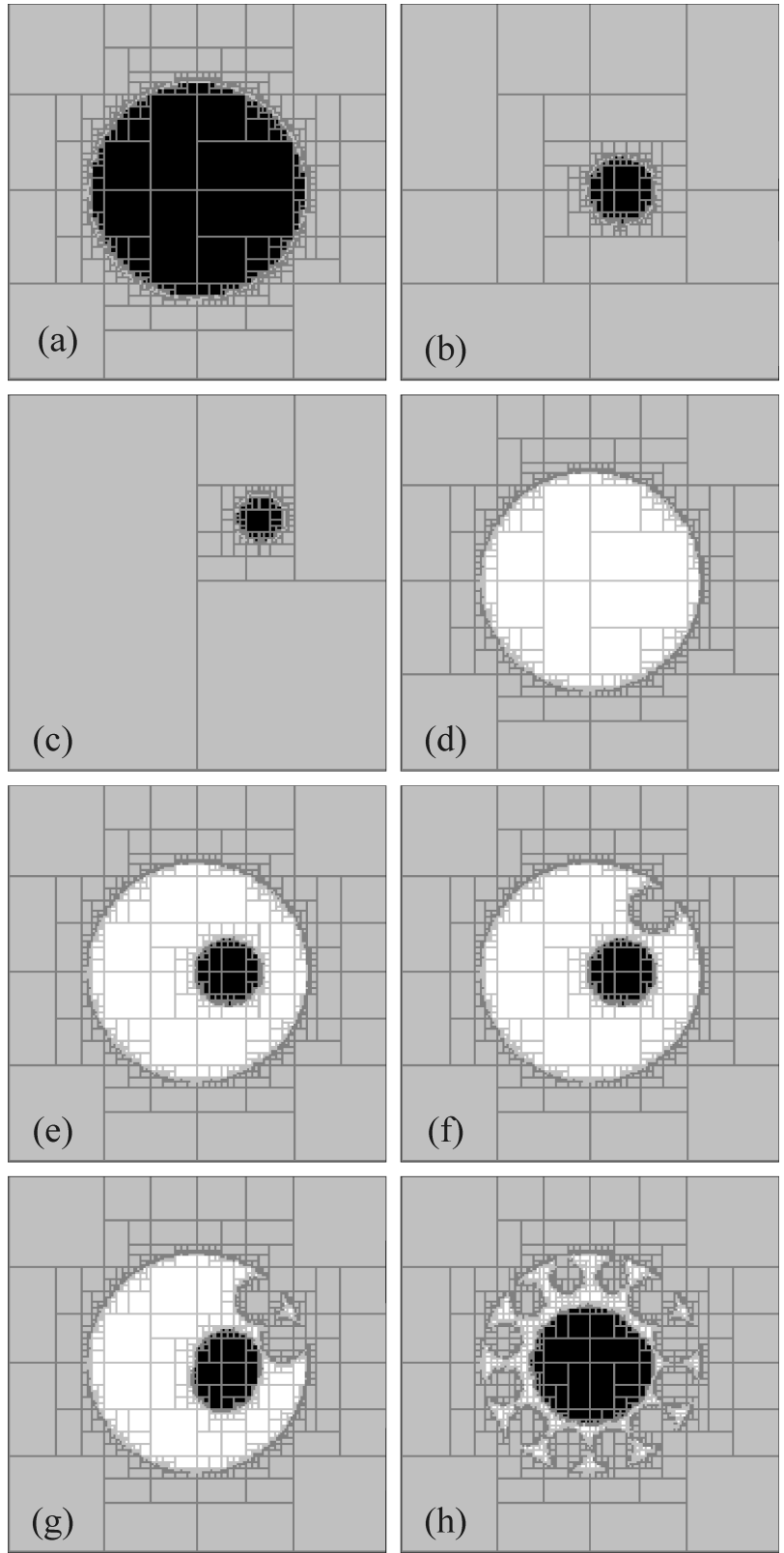

Figure 5: Illustration of the propagation process for set-valued CSP; the frame boxes correspond to $[-3,3]^{2}$ 
[5] D. Daney, N. Andreff, G. Chabert, and Y. Papegay. Interval Method for Calibration of Parallel Robots : Vision-based Experiments. Mechanism and Machine Theory, Elsevier, 41:926-944, 2006.

[6] N. Delanoue, L. Jaulin, and B. Cottenceau. Using interval arithmetic to prove that a set is path-connected. Theoretical Computer Science, Special issue: Real Numbers and Computers, 351(1):119-128, 2006.

[7] C. Gervet. Interval propagation to reason about sets: Definition and implementation of a practical language. Constraints, 1:191-246, 1997.

[8] A. Goldsztejn and L. Jaulin. Inner and outer approximations of existentially quantified equality constraints. In Proceedings of the Twelfth International Conference on Principles and Practice of Constraint Programming, (CP 2006), Nantes (France), 2006.

[9] L. Jaulin, M. Kieffer, O. Didrit, and E. Walter. Applied Interval Analysis, with Examples in Parameter and State Estimation, Robust Control and Robotics. Springer-Verlag, London, 2001.

[10] M. Kieffer, L. Jaulin, I. Braems, and E. Walter. Scientific Computing, Validated Numerics, Interval Methods, Proceedings of SCAN 2000, chapter Guaranteed Set Computation with Subpavings, pages 167-178. Kluwer Academic Publishers, 2001.

[11] S. Lagrange, N. Delanoue, and L. Jaulin. On sufficient conditions of injectivity, development of a numerical test via interval analysis. Reliable computing, 13(5):409-421, 2007.

[12] J. J. Leonard and H. F. Durrant-Whyte. Directed Sonar Sensing for Mobile Robot Navigation. Kluwer, Boston, 1992.

[13] R. E. Moore. Methods and Applications of Interval Analysis. SIAM, Philadelphia, PA, 1979.

[14] N. Ramdani and P.Poignet. Robust dynamic experimental identification of robots with set membership uncertainty. IEEE/ASME Transactions on Mechatronics, 10(2):253Ü-256, 2005.

[15] N. Revol, K. Makino, and M. Berz. Taylor models and floating-point arithmetic: proof that arithmetic operations are validated in COSY. Journal of Logic and Algebraic Programming, 64:135-154, 2005.

[16] S. Thrun, W. Bugard, and D. Fox. Probabilistic Robotics. MIT Press, Cambridge, M.A., 2005.

[17] W. Tucker. The Lorenz attractor exists. Compte Rendu le l'académique des Sciences, 328:1197-1202, 1999. 
[18] J. R. V. Kreinovich, A.V. Lakeyev and P. Kahl. Computational Complexity and Feasibility of Data Processing and Interval Computations. (Applied Optimization). Springer, 1997.

[19] M. van Emden. Algorithmic power from declarative use of redundant constraints. Constraints, 4(4):363-381, 1999. 